\title{
NON-NILPOTENT COMPLEX GEOMETRY OF NILMANIFOLDS AND HETEROTIC SUPERSYMMETRY*
}

\author{
LUIS UGARTE ${ }^{\dagger}$ AND RAQUEL VILLACAMPA
}

\begin{abstract}
We classify non-nilpotent complex structures on 6-nilmanifolds and their associated invariant balanced metrics. As an application we find a large family of solutions of the heterotic supersymmetry equations with non-zero flux, non-flat instanton and constant dilaton satisfying the anomaly cancellation condition with respect to the Chern connection.
\end{abstract}

Key words. Complex structure, Chern connection, balanced Hermitian metric, Strominger system.

AMS subject classifications. 53C55, 53C29, 81T30.

1. Introduction. The complex geometry of nilmanifolds provides a rich source of explicit examples of compact complex manifolds admitting additional special structures with interesting properties. Many authors have studied several aspects of this geometry from different points of view (see for example $[5,6,7,8,14,15,17]$ and the references therein). Here we consider the case when the invariant complex structure is not nilpotent, in the sense of [5], and the nilmanifold has dimension six. This is the lowest dimension when these structures appear. In Section 2 we prove that there exist only 4 non-nilpotent complex structures $J_{\epsilon}^{ \pm}(\epsilon=0,1)$ up to equivalence. According to $[15,17]$ there are, up to isomorphism, only two 6-nilmanifolds admitting non-nilpotent complex structures, although only one of them admits special Hermitian metrics. In fact, we show that if $\epsilon=0$ then the Lie algebra underlying the nilmanifold is isomorphic to $(0,0,0,12,23,14-35)$, whereas if $\epsilon=1$ then the corresponding Lie algebra is $(0,0,12,13,23,14+25)$.

Along this paper $N$ denotes a nilmanifold with $(0,0,0,12,23,14-35)$ as underlying Lie algebra. It turns out that any invariant complex structure on the manifold $N$ is equivalent to $J_{0}^{+}$or $J_{0}^{-}$, and admits Hermitian structures which are balanced in the sense of [13]. Our goal in this paper is the study of the balanced Hermitian geometry of $N$ and its application to the heterotic supersymmetry theory with fluxes.

Strominger investigated in [16] the heterotic superstring background with spacetime supersymmetry and proposed a model based on Hermitian 6-manifolds with holomorphically trivial canonical bundle which are Calabi-Yau with torsion, i.e. the holonomy of the Bismut [2] connection $\nabla^{+}$is reduced to $\mathrm{SU}(3)$. More concretely, as it is described in Section 3, the Strominger system in heterotic string theory consists of the gravitino, dilatino and gaugino equations, together with the anomaly cancellation condition. Given an $\mathrm{SU}(3)$-structure $(J, F, \Psi)$ on $M$, where $F$ denotes the fundamental form, the gravitino and dilatino equations are both satisfied if and only if the holonomy of $\nabla^{+}$reduces to $\mathrm{SU}(3)$, the structure $J$ is integrable and the Lee form $\theta=2 d \phi, \phi$ being the dilaton function (see [9] for general necessary conditions to solve the gravitino equation). The vanishing of the gaugino variation requires a 2form $\Omega^{A} \neq 0$ of instanton type [16], i.e. a Donaldson-Uhlenbeck-Yau SU(3)-instanton

\footnotetext{
*Received March 14, 2012; accepted for publication November 21, 2012.

†Departamento de Matemáticas-I.U.M.A., Universidad de Zaragoza, Campus Plaza San Francisco, 50009 Zaragoza, Spain (ugarte@unizar.es).

$\ddagger$ Centro Universitario de la Defensa-I.U.M.A., Academia General Militar, Crta. de Huesca s/n. 50090 Zaragoza, Spain (raquelvg@unizar.es).
} 
$A$ with non-zero curvature $\Omega^{A}$. Finally, the anomaly cancellation condition in the Strominger system reads as

$$
d T=\frac{\alpha^{\prime}}{4}\left(\operatorname{tr} \Omega \wedge \Omega-\operatorname{tr} \Omega^{A} \wedge \Omega^{A}\right), \quad \alpha^{\prime}>0
$$

where $T=J d F$ is the torsion of $\nabla^{+}$. Here $\Omega$ is the curvature of some metric connection and there are several proposals for it. In $[1,10,16]$ the curvature of the Chern connection $\nabla^{c}$ is proposed to investigate the anomaly cancellation and, based on a construction in [11], Fu and Yau first proved the existence of solutions of the Strominger system on a Hermitian non-Kähler manifold given as a $\mathbb{T}^{2}$-bundle over a $K 3$ surface [10]. In contrast, torus bundles over a complex torus cannot solve the system with respect to the Chern connection in the anomaly cancellation condition [1].

The solutions given in [10] have non-constant dilaton function. If one requires the dilaton to be constant then the Hermitian structure must be balanced. An interesting fact is that for nilmanifolds with invariant Hermitian structure the holonomy of $\nabla^{+}$reduces to $\mathrm{SU}(3)$ if and only if the structure is balanced [8], that is to say, the gravitino equation is equivalent to the dilatino equation with constant dilaton. The classification of 6-nilmanifolds admitting invariant balanced Hermitian structure is given in [17], and it follows from [14, Theorem 4.4] that the corresponding complex nilmanifold is always a torus bundle over a complex 2-torus, except for the nilmanifold $N$. Therefore, according to the result of [1] mentioned above, in order to find a 6-nilmanifold solving the heterotic supersymmetry equations with non-zero flux $T$, constant dilaton and with respect to $\nabla^{c}$ in the anomaly cancellation, we are necessarily led to investigate the non-nilpotent complex geometry of $N$ and their associated invariant balanced metrics.

In Section 4 we find many explicit solutions of the Strominger system; actually, we prove that for any non-nilpotent complex structure $J$ on $N$, the compact complex manifold $(N, J)$ admits a family of solutions of the heterotic supersymmetry equations with non-zero flux, non-flat instanton $A$, i.e. $\Omega^{A} \neq 0$, and constant dilaton satisfying the anomaly cancellation condition with respect to the Chern connection. Some of these solutions are a deformation of the particular solution given in [6] for which the instanton is flat (see Remark 4.3 for details).

The paper is structured as follows. In Section 2 we first prove that any nonnilpotent complex structure $J$ in six dimensions is equivalent to $J_{\epsilon}^{ \pm}(\epsilon=0,1)$, and it has balanced Hermitian metrics if and only if $\epsilon=0$, i.e. $J$ lives on the nilmanifold $N$. Moreover, in Theorem 2.10 we describe the space of invariant balanced $J$-Hermitian structures on $N$ : any such a structure is isomorphic to $\left(J_{0}^{ \pm}, F\right)$, where $F$ belongs to one of two families to which we refer as Family I and Family II. After recalling the heterotic supersymmetry system with constant dilaton, we find in Section 3 an adapted basis for each balanced Hermitian structure in the Families I and II. These adapted bases are used in Section 4 to explicitly construct a 3-parametric family of instantons $A_{\lambda, \mu, \tau}$ for Family I such that $\operatorname{tr}\left(\Omega^{A_{\lambda, \mu, \tau}} \wedge \Omega^{A_{\lambda, \mu, \tau}}\right) \neq 0$ if and only if the parameter $\tau$ is non-zero. In Theorem 4.2 we find many solutions in Family I of the heterotic supersymmetry equations with non-zero flux, non-flat instanton and constant dilaton with respect to the Chern connection in the anomaly cancellation condition. For Family II we also get in Theorem 4.4 many solutions to the supersymmetry system, although in this case the instantons we find become flat. Finally, in Section 4.3 we show an explicit realization of the nilmanifold $N$ and of the balanced Hermitian structures involved. 
2. Non-nilpotent complex structures on 6-nilmanifolds and compatible balanced metrics. Let $M$ be a nilmanifold, i.e. a compact quotient of a simplyconnected nilpotent Lie group $G$ by a lattice $\Gamma$ of maximal rank. Any left-invariant complex structure on $G$ descends to $M$ in a natural way, so a source (possibly empty) of complex structures on $M$ is given by the endomorphisms $J: \mathfrak{g} \longrightarrow \mathfrak{g}$ of the Lie algebra $\mathfrak{g}$ of $G$ such that $J^{2}=-$ Id and satisfying the "Nijenhuis condition"

$$
[J X, J Y]=J[J X, Y]+J[X, J Y]+[X, Y],
$$

for any $X, Y \in \mathfrak{g}$. We shall refer to any such an endomorphism as a complex structure on the Lie algebra $\mathfrak{g}$.

Associated to a complex structure $J$, there exists an ascending series $\left\{\mathfrak{g}_{l}^{J}\right\}_{l \geq 0}$ on the Lie algebra, defined inductively by

$$
\mathfrak{g}_{0}^{J}=\{0\}, \quad \mathfrak{g}_{l}^{J}=\left\{X \in \mathfrak{g} \mid[X, \mathfrak{g}] \subseteq \mathfrak{g}_{l-1}^{J} \quad \text { and }[J X, \mathfrak{g}] \subseteq \mathfrak{g}_{l-1}^{J}\right\}, \quad l \geq 1 .
$$

For any $l \geq 0$, the term $\mathfrak{g}_{l}^{J}$ is a $J$-invariant ideal of $\mathfrak{g}$ which is contained in the term $\mathfrak{g}_{l}=\left\{X \in \mathfrak{g} \mid[X, \mathfrak{g}] \subseteq \mathfrak{g}_{l-1}\right\}$ of the usual ascending central series of $\mathfrak{g}$. But whereas $\mathfrak{g}_{l}$ always reaches the whole Lie algebra $\mathfrak{g}$ when $\mathfrak{g}$ is nilpotent, the series $\left\{\mathfrak{g}_{l}^{J}\right\}_{l \geq 0}$ can stabilize in a proper $J$-ideal of $\mathfrak{g}$. This motivates the following terminology: If $\mathfrak{g}_{l}^{J}=\mathfrak{g}$ for some $l$, then the complex structure $J$ is called nilpotent [5]; otherwise we shall say that $J$ is non-nilpotent.

Definition 2.1. A nilpotent (resp. non-nilpotent) complex structure on a nilmanifold $M$ is a complex structure on $M$ coming from a nilpotent (resp. nonnilpotent) complex structure $J$ on the underlying Lie algebra $\mathfrak{g}$.

Let us denote by $\mathfrak{g}_{\mathbb{C}}$ the complexification of $\mathfrak{g}$ and by $\mathfrak{g}_{\mathbb{C}}^{*}$ its dual. Given an endomorphism $J: \mathfrak{g} \longrightarrow \mathfrak{g}$ such that $J^{2}=-\mathrm{Id}$, we denote by $\mathfrak{g}^{1,0}$ and $\mathfrak{g}^{0,1}$ the eigenspaces of the eigenvalues $\pm i$ of $J$ as an endomorphism of $\mathfrak{g}_{C}^{*}$, respectively. Then, the decomposition $\mathfrak{g}_{\mathbb{C}}^{*}=\mathfrak{g}^{1,0} \oplus \mathfrak{g}^{0,1}$ induces a natural bigraduation on the complexified exterior algebra $\bigwedge^{*} \mathfrak{g}_{\mathbb{C}}^{*}=\oplus_{p, q} \bigwedge^{p, q}\left(\mathfrak{g}^{*}\right)=\oplus_{p, q} \Lambda^{p}\left(\mathfrak{g}^{1,0}\right) \otimes \Lambda^{q}\left(\mathfrak{g}^{0,1}\right)$. If $d$ denotes the usual Chevalley-Eilenberg differential of the Lie algebra, we shall also denote by $d$ its extension to the complexified exterior algebra, i.e. $d: \bigwedge^{*} \mathfrak{g}_{\mathbb{C}}^{*} \longrightarrow \bigwedge^{*+1} \mathfrak{g}_{\mathbb{C}}^{*}$. It is well-known that the endomorphism $J$ is a complex structure if and only if $d\left(\mathfrak{g}^{1,0}\right) \subset$ $\bigwedge^{2,0}\left(\mathfrak{g}^{*}\right) \oplus \bigwedge^{1,1}\left(\mathfrak{g}^{*}\right)$. In the case of nilpotent Lie algebras $\mathfrak{g}$, Salamon proves in [15] the following equivalent condition for the endomorphism $J$ to be a complex structure: $J$ is a complex structure on $\mathfrak{g}$ if and only if $\mathfrak{g}^{1,0}$ has a basis $\left\{\omega^{j}\right\}_{j=1}^{n}$ such that $d \omega^{1}=0$ and

$$
d \omega^{j} \in \mathcal{J}\left(\omega^{1}, \ldots, \omega^{j-1}\right), \quad \text { for } j=2, \ldots, n,
$$

where $\mathfrak{J}\left(\omega^{1}, \ldots, \omega^{j-1}\right)$ is the ideal in $\bigwedge^{*} \mathfrak{g}_{\mathbb{C}}^{*}$ generated by $\left\{\omega^{1}, \ldots, \omega^{j-1}\right\}$. From now on, we shall denote $\omega^{j} \wedge \omega^{k}$ and $\omega^{j} \wedge \overline{\omega^{k}}$ simply by $\omega^{j k}$ and $\omega^{j \bar{k}}$, respectively.

In dimension six, the non-nilpotent complex structures are characterized as follows:

Proposition 2.2. [17, Proposition 2 (a)] Let $J$ be a non-nilpotent complex structure on a nilpotent Lie algebra $\mathfrak{g}$ of dimension 6. Then, there is a basis $\left\{\omega^{j}\right\}_{j=1}^{3}$ for $\mathfrak{g}^{1,0}$ such that

$$
\left\{\begin{array}{l}
d \omega^{1}=0 \\
d \omega^{2}=E \omega^{13}+\omega^{1 \overline{3}} \\
d \omega^{3}=A \omega^{1 \overline{1}}+i b \omega^{1 \overline{2}}-i b \bar{E} \omega^{2 \overline{1}}
\end{array}\right.
$$


where $A, E \in \mathbb{C}$ with $|E|=1$, and $b \in \mathbb{R}-\{0\}$.

A complex structure $J$ on $\mathfrak{g}$ is said to be equivalent to a complex structure $J^{\prime}$ on $\mathfrak{g}^{\prime}$ if there is an isomorphism $A: \mathfrak{g} \longrightarrow \mathfrak{g}^{\prime}$ of Lie algebras such that $J^{\prime} A=A J$. Equivalently, the linear isomorphism $A^{*}: \mathfrak{g}^{\prime *} \longrightarrow \mathfrak{g}^{*}$ commutes with the ChevalleyEilenberg differentials and the extension of $A^{*}$ to the complexified exterior algebra preserves the bigraduations induced by $J$ and $J^{\prime}$.

In the next result we prove that up to equivalence there are only four non-nilpotent complex structures in dimension six.

Proposition 2.3. Let $J$ be a non-nilpotent complex structure on a 6-dimensional nilpotent Lie algebra $\mathfrak{g}$. Then, there is a $(1,0)$-basis $\left\{\omega^{j}\right\}_{j=1}^{3}$ satisfying

$$
\left\{\begin{aligned}
d \omega^{1} & =0 \\
d \omega^{2} & =\omega^{13}+\omega^{1 \overline{3}} \\
d \omega^{3} & =i \epsilon \omega^{1 \overline{1}} \pm i\left(\omega^{1 \overline{2}}-\omega^{2 \overline{1}}\right)
\end{aligned}\right.
$$

with $\epsilon=0,1$. The four complex structures $J_{\epsilon}^{ \pm}(\epsilon=0,1)$ corresponding to the different choices of the coefficients in (2.2) are not equivalent.

Proof. From Proposition 2.2 we have the existence of a $(1,0)$-basis $\left\{\omega^{\prime \prime j}\right\}_{j=1}^{3}$ satisfying

$$
d \omega^{\prime \prime 1}=0, \quad d \omega^{\prime \prime 2}=E \omega^{\prime \prime 13}+\omega^{\prime \prime 1 \overline{3}}, \quad d \omega^{\prime \prime 3}=A \omega^{\prime \prime 1 \overline{1}}+i b \omega^{\prime \prime 1 \overline{2}}-i b \bar{E} \omega^{\prime \prime 2 \overline{1}},
$$

where $A, E \in \mathbb{C}$ with $|E|=1$ and $b \in \mathbb{R}-\{0\}$. The coefficients of these equations are easily seen to be reduced to $E=1$ and $b= \pm 1$ when we consider the new (1,0)basis $\left\{\omega^{\prime 1}=\sqrt{|b|} \omega^{\prime \prime 1}, \omega^{\prime 2}=\bar{\vartheta} \sqrt{|b|} \omega^{\prime \prime 2}, \omega^{\prime 3}=\vartheta \omega^{\prime \prime 3}\right\}, \vartheta$ being a non-zero solution of $\bar{\vartheta} E=\vartheta$. In fact, in terms of the basis $\left\{\omega^{\prime j}\right\}_{j=1}^{3}$ the structure equations become

$$
d \omega^{\prime 1}=0, \quad d \omega^{\prime 2}=\omega^{\prime 13}+\omega^{\prime 1 \overline{3}}, \quad d \omega^{\prime 3}=B \omega^{\prime 1 \overline{1}} \pm i\left(\omega^{\prime 1 \overline{2}}-\omega^{\prime 2 \overline{1}}\right),
$$

for some $B \in \mathbb{C}$. Let us denote $x=\mathfrak{R e} B$ and $y=\mathfrak{I m} B$. Now, if $y \neq 0$ then with respect to the new $(1,0)$-basis $\left\{\omega^{1}=i \omega^{1}, \omega^{2}=\mp \frac{x}{2 y} \omega^{11}+\frac{i}{y} \omega^{\prime 2}, \omega^{3}=\frac{1}{y} \omega^{\prime 3}\right\}$ the equations above reduce to (2.2) with $\epsilon=1$. In other case, if $B=x \in \mathbb{R}-\{0\}$ then we get (2.2) with $\epsilon=0$ with respect to $\left\{\omega^{1}=i \omega^{\prime 1}, \omega^{2}= \pm \omega^{\prime 1}-\frac{2 i}{x} \omega^{\prime 2}, \omega^{3}=-\frac{2}{x} \omega^{\prime 3}\right\}$.

Finally, let us consider $\left\{\omega^{j}\right\}_{j=1}^{3}$ and $\left\{\sigma^{j}\right\}_{j=1}^{3}(1,0)$-bases corresponding to two complex structures given by $(2.2)$, i.e.

$$
\begin{aligned}
& d \omega^{1}=0, \quad d \omega^{2}=\omega^{13}+\omega^{1 \overline{3}}, \quad d \omega^{3}=i \epsilon \omega^{1 \overline{1}}+i c\left(\omega^{1 \overline{2}}-\omega^{2 \overline{1}}\right), \\
& d \sigma^{1}=0, \quad d \sigma^{2}=\sigma^{13}+\sigma^{1 \overline{3}}, \quad d \sigma^{3}=i \epsilon^{\prime} \sigma^{1 \overline{1}}+i c^{\prime}\left(\sigma^{1 \overline{2}}-\sigma^{2 \overline{1}}\right),
\end{aligned}
$$

where $\epsilon, \epsilon^{\prime} \in\{0,1\}$ and $c, c^{\prime} \in\{ \pm 1\}$. If we express $\sigma^{i}=m_{i 1} \omega^{1}+m_{i 2} \omega^{2}+m_{i 3} \omega^{3}$ for $i=1,2,3$ and $\left(m_{i j}\right) \in \mathrm{GL}(3, \mathbb{C})$, then $d \sigma^{i}=m_{i 1} d \omega^{1}+m_{i 2} d \omega^{2}+m_{i 3} d \omega^{3}$ is equivalent to

$$
\begin{gathered}
m_{12}=m_{13}=m_{23}=m_{31}=m_{32}=0, \\
m_{33} \in \mathbb{R}-\{0\}, \quad \mathfrak{I m}\left(m_{11} \overline{m_{21}}\right)=0, \quad m_{33} c=m_{11} \overline{m_{22}} c^{\prime} \\
m_{11} m_{33}=m_{22}, \quad m_{33} \epsilon=\left|m_{11}\right|^{2} \epsilon^{\prime} .
\end{gathered}
$$


These last conditions imply that $\epsilon=\epsilon^{\prime}$ and $c=\left|m_{11}\right|^{2} c^{\prime}$, so necessarily $c=c^{\prime}$. In conclusion, there does not exist any equivalence between any two of the complex structures (2.2).

The classification of 6-dimensional nilpotent Lie algebras admitting non-nilpotent complex structure is as follows:

Proposition 2.4. If $\epsilon=0$ in (2.2) then the Lie algebra $\mathfrak{g}$ is isomorphic to $(0,0,0,12,23,14-35)$, whereas if $\epsilon=1$ then $\mathfrak{g} \cong(0,0,12,13,23,14+25)$.

Proof. For the case $\epsilon=0$ it suffices to consider the real basis $\left\{\alpha^{1}, \ldots, \alpha^{6}\right\}$ for $\mathfrak{g}^{*}$ given by $\alpha^{1}-i \alpha^{3}=\omega^{1}, \alpha^{4}+i \alpha^{5}=\omega^{2}$ and $\frac{1}{2} \alpha^{2} \pm 2 i \alpha^{6}=\omega^{3}$. For $\epsilon=1$ it suffices to consider the basis $\left\{\alpha^{1}, \ldots, \alpha^{6}\right\}$ for $\mathfrak{g}^{*}$ given by $\frac{\sqrt{2}}{2}\left(\alpha^{1}+i \alpha^{2}\right)=\omega^{1}, \sqrt{2}\left(\alpha^{4}+i \alpha^{5}\right)=\omega^{2}$ and $\alpha^{3} \pm 2 i \alpha^{6}=\omega^{3}$.

In the next result we summarize the previous propositions.

Corollary 2.5. The Lie algebras $(0,0,0,12,23,14-35)$ and $(0,0,12,13,23,14+$ $25)$ are the only 6-dimensional nilpotent Lie algebras admitting non-nilpotent complex structure. Moreover, up to equivalence, each of these Lie algebras has only two complex structures.

REMARK 2.6. If a 6-dimensional nilpotent Lie algebra $\mathfrak{g}$ has a nilpotent complex structure, then all the complex structures on $\mathfrak{g}$ must be nilpotent. As it is proved in [17, Corollary 7], this assertion follows from the fact that the first term $\mathfrak{g}_{1}^{J}$ in the series adapted to $J$, which is a $J$-invariant ideal of $\mathfrak{g}$ contained in the center, is non-trivial; therefore, such a Lie algebra $\mathfrak{g}$ is not isomorphic neither to $(0,0,0,12,23,14-35)$ nor to $(0,0,12,13,23,14+25)$ because they have 1-dimensional center. This result, together with the classification of 6-dimensional nilpotent Lie algebras admitting nilpotent complex structure obtained in [4], provides the classification of 6-dimensional nilpotent Lie algebras admitting complex structure, given by Salamon in [15].

REMARK 2.7. As it is observed in [15], the Lie algebras $(0,0,0,12,23,14-35)$ and $(0,0,12,13,23,14+25)$ correspond to $\mathfrak{h}_{19}^{-}$and $\mathfrak{h}_{26}^{+}$, respectively, in the notation given in [4].

Now, let $\mathfrak{g}$ be a Lie algebra of dimension 6. A Hermitian structure on $\mathfrak{g}$ is a pair $(J, g)$, where $J$ is a complex structure on $\mathfrak{g}$ and $g$ is an inner product on $\mathfrak{g}$ compatible with $J$ in the usual sense, i.e. $g(\cdot, \cdot)=g(J \cdot, J \cdot)$. The associated fundamental form $F \in \bigwedge^{2} \mathfrak{g}^{*}$ is defined by $F(X, Y)=g(X, J Y)$ and expresses in terms of any basis $\left\{\omega^{j}\right\}_{j=1}^{3}$, of type $(1,0)$ with respect to $J$, by

(2.3) $2 F=i\left(r^{2} \omega^{1 \overline{1}}+s^{2} \omega^{2 \overline{2}}+t^{2} \omega^{3 \overline{3}}\right)+u \omega^{1 \overline{2}}-\bar{u} \omega^{2 \overline{1}}+v \omega^{2 \overline{3}}-\bar{v} \omega^{3 \overline{2}}+z \omega^{1 \overline{3}}-\bar{z} \omega^{3 \overline{1}}$,

for some $r, s, t \in \mathbb{R}$ and $u, v, z \in \mathbb{C}$. Since we are using the convention $(J \alpha)(X)=$ $-\alpha(J X)$ for $\alpha \in \mathfrak{g}^{*}$, the inner product $g$ is given by

$$
\begin{aligned}
g= & r^{2} \omega^{1} \omega^{\overline{1}}+s^{2} \omega^{2} \omega^{\overline{2}}+t^{2} \omega^{3} \omega^{\overline{3}} \\
& -\frac{i}{2}\left(u \omega^{1} \omega^{\overline{2}}-\bar{u} \omega^{2} \omega^{\overline{1}}+v \omega^{2} \omega^{\overline{3}}-\bar{v} \omega^{3} \omega^{\overline{2}}+z \omega^{1} \omega^{\overline{3}}-\bar{z} \omega^{3} \omega^{\overline{1}}\right) .
\end{aligned}
$$

Here $\omega^{j} \omega^{\bar{k}}=\frac{1}{2}\left(\omega^{j} \otimes \omega^{\bar{k}}+\omega^{\bar{k}} \otimes \omega^{j}\right)$ denotes the symmetric product of $\omega^{j}$ and $\omega^{\bar{k}}$. Notice that the positive definiteness of $g$ implies that the coefficients $r^{2}, s^{2}, t^{2}$ are 
non-zero real numbers and $u, v, z \in \mathbb{C}$ satisfy $r^{2} s^{2}>|u|^{2}, s^{2} t^{2}>|v|^{2}, r^{2} t^{2}>|z|^{2}$ and $r^{2} s^{2} t^{2}+2 \mathfrak{R e}(i \bar{u} \bar{v} z)>t^{2}|u|^{2}+r^{2}|v|^{2}+s^{2}|z|^{2}$.

Fixed $J$, since $g$ and $F$ are mutually determined by each other, we shall also denote the Hermitian structure $(J, g)$ by the pair $(J, F)$. Recall that the Hermitian structure $(J, F)$ is said to be balanced if $F^{2}$ is a closed form or, equivalently, $F \wedge d F=0$. Our goal in this section is to classify the balanced Hermitian structures $(J, F)$ on 6 dimensional nilpotent Lie algebras $\mathfrak{g}$ with $J$ non-nilpotent, up to equivalence. We recall that a Hermitian structure $(J, F)$ on $\mathfrak{g}$ is said to be equivalent to a Hermitian structure $\left(J^{\prime}, F^{\prime}\right)$ on $\mathfrak{g}^{\prime}$ if there is an isomorphism $A: \mathfrak{g} \longrightarrow \mathfrak{g}^{\prime}$ of Lie algebras such that $J^{\prime} A=A J$ and $F=A^{*} F^{\prime}$. Notice that this implies that $g=A^{*} g^{\prime}$, where $g$ and $g^{\prime}$ denote the inner products associated to $(J, F)$ and $\left(J^{\prime}, F^{\prime}\right)$, respectively.

The only 6-dimensional nilpotent Lie algebra admitting balanced Hermitian metric with respect to a non-nilpotent complex structure is $(0,0,0,12,23,14-35)$, which from now on we shall denote it by $\mathfrak{n}$ instead of $\mathfrak{h}_{19}^{-}$. Next we describe the balanced Hermitian metrics on $\mathfrak{n}$ with respect to a (1,0)-basis satisfying (2.2) for $\epsilon=0$.

Proposition 2.8. If $\mathfrak{g}$ is a 6-dimensional nilpotent Lie algebra admitting balanced Hermitian structure $(J, F)$ with respect to a non-nilpotent complex structure $J$, then $\mathfrak{g} \cong \mathfrak{n}=(0,0,0,12,23,14-35)$. Moreover, there is a $(1,0)$-basis $\left\{\omega^{j}\right\}_{j=1}^{3}$ satisfying

$$
d \omega^{1}=0, \quad d \omega^{2}=\omega^{13}+\omega^{1 \overline{3}}, \quad d \omega^{3}= \pm i\left(\omega^{1 \overline{2}}-\omega^{2 \overline{1}}\right),
$$

such that the fundamental form $F$ is given by

$$
2 F=i\left(r^{2} \omega^{1 \overline{1}}+s^{2} \omega^{2 \overline{2}}+t^{2} \omega^{3 \overline{3}}\right)+u \omega^{1 \overline{2}}-\bar{u} \omega^{2 \overline{1}}+v \omega^{2 \overline{3}}-\bar{v} \omega^{3 \overline{2}}+z \omega^{1 \overline{3}}-\bar{z} \omega^{3 \overline{1}},
$$

with

$$
u+\bar{u}=0, \quad z=-i u v / s^{2}
$$

Proof. From [17, Proposition 25 (a)] we have that if $J$ is a non-nilpotent complex structure defined by $(2.2)$, then $(J, F)$ is balanced if and only if $i \epsilon s^{2} \pm(u+\bar{u})=0$ and $z=-i u v / s^{2}$, which is equivalent to the conditions

$$
\epsilon=0, \quad u+\bar{u}=0 \quad \text { and } \quad z=-i u v / s^{2} .
$$

The result follows from Proposition 2.4.

Notice that as a consequence of this result any complex structure $J$ on $\mathfrak{n}$ admits balanced $J$-Hermitian structures. Next we classify balanced Hermitian structures on the Lie algebra $\mathfrak{n}$ up to equivalence. First, we need the following result which describes the automorphisms of the Lie algebra $\mathfrak{n}$ preserving $J_{0}^{+}$or $J_{0}^{-}$.

Lemma 2.9. Let $\left\{\omega^{j}\right\}_{j=1}^{3}$ be a $(1,0)$-basis satisfying (2.5). Then, the transformations given by $\left\{\omega^{1}=e^{i \theta} \omega^{1}, \omega^{\prime 2}=B \omega^{1}+c e^{i \theta} \omega^{2}, \omega^{\prime 3}=c \omega^{3}\right\}$, where $c \in \mathbb{R}-\{0\}$, $\theta \in[0,2 \pi), B \in \mathbb{C}$ such that $\mathfrak{I m}\left(e^{i \theta} \bar{B}\right)=0$, are the only ones preserving the structure equations (2.5).

Proof. It is immediate to check that any basis $\left\{\omega^{\prime j}\right\}_{j=1}^{3}$ satisfies the equations (2.5) if $\left\{\omega^{j}\right\}_{j=1}^{3}$ does. On the other hand, consider $\left\{\omega^{j}\right\}_{j=1}^{3}$ and $\left\{\omega^{\prime j}\right\}_{j=1}^{3}$ satisfying (2.5) for the same choice of sign. If we write $\omega^{i}=m_{i 1} \omega^{1}+m_{i 2} \omega^{2}+m_{i 3} \omega^{3}$, for $i=1,2,3$, 
with $\left(m_{i j}\right) \in \mathrm{GL}(3, \mathbb{C})$, then it is easy to see that $d \omega^{\prime i}=m_{i 1} d \omega^{1}+m_{i 2} d \omega^{2}+m_{i 3} d \omega^{3}$ is equivalent to

$$
\begin{gathered}
m_{12}=m_{13}=m_{23}=m_{31}=m_{32}=0, \quad \mathfrak{I m}\left(m_{11} \overline{m_{21}}\right)=0, \\
m_{33} \in \mathbb{R}-\{0\}, \quad m_{11} m_{33}=m_{22}, \quad m_{11} \overline{m_{22}}=m_{33} .
\end{gathered}
$$

The last two conditions imply $\left|m_{11}\right|^{2}=1$, so $m_{11}=e^{i \theta}$. If we put $m_{33}=c$, then $m_{22}=c e^{i \theta}$ and finally, $\mathfrak{I m}\left(e^{i \theta} \overline{m_{21}}\right)=0$.

Theorem 2.10. Let $(J, F)$ be a balanced Hermitian structure on $\mathfrak{n}$ for $J=J_{0}^{ \pm}$. Then, in the conditions of Proposition 2.8, the fundamental form $F$ is equivalent to one and only one form in the following families:

Family I: $\quad 2 F=i\left(r^{2} \omega^{1 \overline{1}}+s^{2} \omega^{2 \overline{2}}+\omega^{3 \overline{3}}\right), \quad r, s \neq 0$;

Family II: $\quad 2 F=i\left(r^{2} \omega^{1 \overline{1}}+s^{2} \omega^{2 \overline{2}}+t^{2} \omega^{3 \overline{3}}\right)+\omega^{2 \overline{3}}-\omega^{3 \overline{2}}, \quad r \neq 0, \quad s^{2} t^{2}>1$.

Proof. Let $\left\{\sigma^{j}\right\}_{j=1}^{3}$ be a $(1,0)$-basis satisfying $(2.5)$ and let us consider a general compatible metric (2.4) satisfying the balanced conditions (2.7). Let us write $v$ as $v=$ $-c^{2} e^{i \theta}=c^{2} e^{i(\theta-\pi)}$. If $v=0$ then taking $\left\{\omega^{1}=-\sigma^{1}, \omega^{2}=t\left(-\frac{u i}{s^{2}} \sigma^{1}+\sigma^{2}\right), \omega^{3}=\right.$ $\left.-t \sigma^{3}\right\}$ we obtain a balanced structure in Family I. On the other hand, if $v \neq 0$ then the basis $\left\{\omega^{1}=-e^{i \theta} \sigma^{1}, \omega^{2}=c e^{i \theta}\left(-\frac{u i}{s^{2}} \sigma^{1}+\sigma^{2}\right), \omega^{3}=-c \sigma^{3}\right\}$ keeps (2.5) by Lemma 2.9 (observe that $\mathfrak{I m}\left(\frac{i u}{s^{2}}\right)=0$ because $u \in i \mathbb{R}$ ) and provides a balanced structure in Family II, concretely

$$
2 F=i\left(\frac{r^{2} s^{2}-|u|^{2}}{s^{2}} \omega^{1 \overline{1}}+\frac{s^{2}}{c^{2}} \omega^{2 \overline{2}}+\frac{t^{2}}{c^{2}} \omega^{3 \overline{3}}\right)+\omega^{2 \overline{3}}-\omega^{3 \overline{2}} .
$$

Finally, we see that the structures in Families I and II are not equivalent. Consider the pairs

$$
\left\{\omega^{1}, \omega^{2}, \omega^{3}\right\}, \quad F_{\omega}=i\left(r^{2} \omega^{1 \overline{1}}+s^{2} \omega^{2 \overline{2}}+t^{2} \omega^{3 \overline{3}}\right)+\varepsilon_{1}\left(\omega^{2 \overline{3}}-\omega^{3 \overline{2}}\right),
$$

and

$$
\left\{\sigma^{1}, \sigma^{2}, \sigma^{3}\right\}, \quad F_{\sigma}=i\left(a^{2} \sigma^{1 \overline{1}}+b^{2} \sigma^{2 \overline{2}}+c^{2} \sigma^{3 \overline{3}}\right)+\varepsilon_{2}\left(\sigma^{2 \overline{3}}-\sigma^{3 \overline{2}}\right),
$$

where $\left\{\omega^{j}\right\}_{j=1}^{3}$ and $\left\{\sigma^{j}\right\}_{j=1}^{3}$ are $(1,0)$-basis satisfying (2.5) for the same complex structure. Let us suppose that there exists an equivalence between them. By Lemma 2.9, $\omega^{1}=e^{i \theta} \sigma^{1}, \omega^{2}=B \sigma^{1}+\lambda e^{i \theta} \sigma^{2}$ and $\omega^{3}=\lambda \sigma^{3}$ for some $\lambda \in \mathbb{R}-\{0\}, \theta \in[0,2 \pi), B \in \mathbb{C}$ such that $\mathfrak{I m}\left(e^{i \theta} \bar{B}\right)=0$. Then, the conditions that transform $F_{\omega}$ into $F_{\sigma}$ are:

$$
i\left(\lambda \bar{B} e^{i \theta}\right)=\varepsilon_{1} B \lambda=0, \quad \varepsilon_{1} \lambda^{2} e^{i \theta}=\varepsilon_{2}, \quad r^{2}+s^{2}|B|^{2}=a^{2}, \quad s^{2} \lambda^{2}=b^{2}, \quad t^{2} \lambda^{2}=c^{2} .
$$

From the first relation we have that $B=0$ and, in consequence, $r^{2}=a^{2}$. Now, if $\varepsilon_{1}=\varepsilon_{2}=1$, then $\lambda^{2}=e^{i \theta}=1$ and therefore, $s^{2}=b^{2}$ and $t^{2}=c^{2}$. If $\varepsilon_{1}=\varepsilon_{2}=0$ and $t^{2}=c^{2}=1$, then $\lambda^{2}=1$ and again $s^{2}=b^{2}$. Finally, if $\varepsilon_{1}=1$ and $\varepsilon_{2}=0, c^{2}=1$, then $\lambda^{2} e^{i \theta}=0$ which is not possible. 
3. Heterotic supersymmetry with constant dilaton. Conformally balanced Hermitian structures are a key ingredient in the solutions of the Strominger system of equations in heterotic string theory [16]. Before writing the system explicitly, we first recall that any Hermitian structure $(J, F)$ on a $2 n$-dimensional manifold $M$ has a unique Hermitian connection with torsion $T$ given by $g(X, T(Y, Z))=$ $J d F(X, Y, Z)=-d F(J X, J Y, J Z), g$ being the associated metric [2]. This torsion connection is known as the Bismut connection of $(J, F)$ and will be denoted here by $\nabla^{+}$. From now on, we shall identify $T$ with the 3 -form $J d F$. In relation to the Levi-Civita connection $\nabla^{g}$ of the Riemannian metric $g$, the Bismut connection is determined by $\nabla^{+}=\nabla^{g}+\frac{1}{2} T$. On the other hand, let $\theta$ be the Lee form associated to the Hermitian structure $(J, F)$, that is, $\theta=\frac{1}{1-n} J \delta F$, where $\delta$ denotes the formal adjoint of $d$ with respect to the associated metric $g$. The 1 -form $\theta$ vanishes identically if and only if the form $F^{n-1}$ is closed, i.e. the Hermitian structure is balanced.

In addition to a conformally balanced Hermitian structure, the Strominger system also requires $M$ to be a manifold of complex dimension 3 endowed with a non-vanishing holomorphic (3,0)-form $\Psi$. Therefore, one is led to consider certain special Hermitian $\mathrm{SU}(3)$-structures $(J, F, \Psi)$ in six dimensions [3].

Here we are interested in finding explicit compact solutions of the heterotic supersymmetry equations with non-zero flux $H=T$. More concretely, in the heterotic theory of superstrings with fluxes one looks for a compact 6 -manifold $M$ endowed with a Hermitian SU(3)-structure $(J, F, \Psi)$ satisfying the following system of equations [16]:

(a) Gravitino equation: the holonomy of the Bismut connection $\nabla^{+}$is contained in $\mathrm{SU}(3)$.

(b) Dilatino equation: the Lee form $\theta$ is exact; moreover $\theta=2 d \phi$, where $\phi$ is the dilaton function.

(c) Gaugino equation: there is a Donaldson-Uhlenbeck-Yau SU(3)-instanton; that is to say, a Hermitian vector bundle of rank $r$ over $M$ equipped with an $\mathrm{SU}(3)$-instanton, i.e. a connection $A$ with curvature 2-form $\Omega^{A} \in \mathfrak{s u}(3)$.

(d) Anomaly cancellation condition:

$$
d T=2 \pi^{2} \alpha^{\prime}\left(p_{1}(\nabla)-p_{1}(A)\right), \quad \alpha^{\prime}>0 .
$$

Here $p_{1}$ denotes the 4 -form representing the first Pontrjagin class of the connection, which is given in terms of the curvature forms $\Omega_{j}^{i}$ of the connection by $p_{1}=\frac{1}{8 \pi^{2}} \operatorname{tr} \Omega \wedge \Omega=\frac{1}{8 \pi^{2}} \sum_{1 \leq i<j \leq 6} \Omega_{j}^{i} \wedge \Omega_{j}^{i}$. The requirement $\alpha^{\prime}>0$ in the anomaly cancellation condition is due to physical considerations. Different connections $\nabla$ in (d) correspond to different regularization schemes in the two-dimensional worldsheet nonlinear sigma model. In $[1,10,16]$ the Chern connection $\nabla^{c}$ is proposed to investigate the anomaly cancellation.

As an application of the results given in Section 2, next we find solutions to the system above in the case when the dilaton is constant, which implies that the structure $(J, g)$ is balanced Hermitian, and satisfying the anomaly cancellation condition with curvature term taken with respect to the Chern connection, i.e. $\nabla=\nabla^{c}$.

We focus on six-dimensional nilmanifolds $M=\Gamma \backslash G$ endowed with an invariant non-nilpotent complex structure $J$. As it is explained in the introduction, this is the appropriate class of structures to look for solutions of the Strominger system with respect the Chern connection in the anomaly cancellation condition. According to [8, Proposition 6.1], for invariant Hermitian metrics on $M$ the balanced condition is equivalent to the reduction of the holonomy group of the associated Bismut connection $\nabla^{+}$to a subgroup of $\mathrm{SU}(3)$, i.e. the conditions (a) and (b) in the system above are 
equivalent. Also, notice that given an invariant Hermitian structure on a nilmanifold any invariant $(3,0)$-form $\Psi$ is closed [15].

Given a Hermitian structure $(J, F)$, we say that a (real) coframe $\left\{e^{1}, \ldots, e^{6}\right\}$ is an adapted basis for $(J, F)$ if $J e^{1}=-e^{2}, J e^{3}=-e^{4}, J e^{5}=-e^{6}$ and $F=e^{12}+e^{34}+e^{56}$. The associated metric $g$ expresses in terms of this basis by $g=e^{1} \otimes e^{1}+\cdots+e^{6} \otimes e^{6}$, i.e. $\left\{e^{1}, \ldots, e^{6}\right\}$ is orthonormal. In the subsequent sections we shall always consider an adapted basis and the (3,0)-form $\Psi$ defining the $\mathrm{SU}(3)$-structure as $\Psi=\left(e^{1}+\right.$ $\left.i e^{2}\right) \wedge\left(e^{3}+i e^{4}\right) \wedge\left(e^{5}+i e^{6}\right)$.

There is a clear advantage when working with adapted bases. For instance, in the gaugino equation the curvature 2 -form $\Omega^{A} \in \mathfrak{s u}(3)$ if and only if

$$
\begin{aligned}
& \left(\Omega^{A}\right)_{j}^{i}\left(e_{1}, e_{2}\right)+\left(\Omega^{A}\right)_{j}^{i}\left(e_{3}, e_{4}\right)+\left(\Omega^{A}\right)_{j}^{i}\left(e_{5}, e_{6}\right)=0, \\
& \left(\Omega^{A}\right)_{j}^{i}\left(J e_{k}, J e_{l}\right)=\left(\Omega^{A}\right)_{j}^{i}\left(e_{k}, e_{l}\right), \quad \forall i, j, k, l,
\end{aligned}
$$

where $\left\{e_{1}, \ldots, e_{6}\right\}$ is the dual basis of a basis $\left\{e^{1}, \ldots, e^{6}\right\}$ adapted to the $\mathrm{SU}(3)$ structure. Moreover, since we are working with invariant structures, the adapted bases are globally defined on the nilmanifold $M$ because they stem from the corresponding Lie group $G$ (actually, from the Lie algebra $\mathfrak{g}$ of $G$ ) by passing to the quotient.

In general, for any 6-dimensional Lie group, let us consider the structure equations with respect to a basis of left-invariant 1-forms:

$$
d e^{k}=\sum_{1 \leq i<j \leq 6} a_{i j}^{k} e^{i j}, \quad k=1, \ldots, 6 .
$$

Let $g=e^{1} \otimes e^{1}+\cdots+e^{6} \otimes e^{6}$ be the Riemannian metric for which the basis $\left\{e^{k}\right\}_{k=1}^{6}$ is orthonormal, and denote by $\left\{e_{1}, \ldots, e_{6}\right\}$ the dual basis.

Given a linear connection $\nabla$, the connection 1-forms $\sigma_{j}^{i}$ with respect to the above basis are

$$
\sigma_{j}^{i}\left(e_{k}\right)=g\left(\nabla_{e_{k}} e_{j}, e_{i}\right)
$$

i.e. $\nabla_{X} e_{j}=\sigma_{j}^{1}(X) e_{1}+\cdots+\sigma_{j}^{6}(X) e_{6}$. The curvature 2 -forms $\Omega_{j}^{i}$ of $\nabla$ are given in terms of the connection 1-forms $\sigma_{j}^{i}$ by

$$
\Omega_{j}^{i}=d \sigma_{j}^{i}+\sum_{1 \leq k \leq 6} \sigma_{k}^{i} \wedge \sigma_{j}^{k}
$$

and the first Pontrjagin class is represented by the 4 -form $p_{1}(\nabla)=\frac{1}{8 \pi^{2}} \sum_{1 \leq i<j \leq 6} \Omega_{j}^{i} \wedge$ $\Omega_{j}^{i}$.

Now, if $J$ is a complex structure compatible with $g$ then, in addition to the Bismut connection $\nabla^{+}=\nabla^{g}+\frac{1}{2} T$, we can also consider the Chern connection $\nabla^{c}$, which is defined by $\nabla^{c}=\nabla^{g}+\frac{1}{2} C$ with $C(\cdot, \cdot, \cdot)=d F(J \cdot, \cdot, \cdot)$, that is

$$
\begin{aligned}
& g\left(\nabla_{X}^{c} Y, Z\right)=g\left(\nabla_{X}^{g} Y, Z\right)+\frac{1}{2} C(X, Y, Z), \\
& C(X, Y, Z)=d F(J X, Y, Z)=-T(X, J Y, J Z) .
\end{aligned}
$$

Observe that $C(X, \cdot, \cdot)=(J X\lrcorner d F)(\cdot, \cdot)$ is a 2-form and $C(\cdot, Y, Z)=(J \cdot\lrcorner d F)(Y, Z)$ a 1 -form. 
Since $d e^{k}\left(e_{i}, e_{j}\right)=-e^{k}\left(\left[e_{i}, e_{j}\right]\right)$, the Levi-Civita connection 1-forms $\left(\sigma^{g}\right)_{j}^{i}$ of the metric $g$ express in terms of the structure constants by

$$
\left(\sigma^{g}\right)_{j}^{i}\left(e_{k}\right)=-\frac{1}{2}\left(g\left(e_{i},\left[e_{j}, e_{k}\right]\right)-g\left(e_{k},\left[e_{i}, e_{j}\right]\right)+g\left(e_{j},\left[e_{k}, e_{i}\right]\right)\right)=\frac{1}{2}\left(a_{j k}^{i}-a_{i j}^{k}+a_{k i}^{j}\right) .
$$

Therefore, the connection 1-forms $\left(\sigma^{c}\right)_{j}^{i}$ for the Chern connection $\nabla^{c}$ are determined by

$$
\left(\sigma^{c}\right)_{j}^{i}\left(e_{k}\right)=\left(\sigma^{g}\right)_{j}^{i}\left(e_{k}\right)-\frac{1}{2} C_{j}^{i}\left(e_{k}\right)=\frac{1}{2}\left(a_{j k}^{i}-a_{i j}^{k}+a_{k i}^{j}\right)-\frac{1}{2} C_{j}^{i}\left(e_{k}\right)
$$

where $C_{j}^{i}$ are the torsion 1 -forms defined by

$$
C_{j}^{i}\left(e_{k}\right)=C\left(e_{k}, e_{i}, e_{j}\right)=d F\left(J e_{k}, e_{i}, e_{j}\right) .
$$

3.1. Adapted basis for balanced Hermitian structures in Families I and II. In this section we find an adapted basis for the balanced Hermitian structures given in Families I and II of Theorem 2.10.

To get an orthonormal coframe $\left\{e^{1}, \ldots, e^{6}\right\}$ with respect to a metric in Family I it is enough to consider

$$
e^{1}+i e^{2}=r \omega^{1}, \quad e^{3}+i e^{4}=s \omega^{2}, \quad e^{5}+i e^{6}=\omega^{3} .
$$

In terms of this basis, the structure equations become

$$
\left\{\begin{aligned}
d e^{1} & =d e^{2}=d e^{5}=0, \\
d e^{3} & =\frac{2 s}{r} e^{15} \\
d e^{4} & =\frac{2 s}{r} e^{25} \\
d e^{6} & = \pm \frac{2}{r s}\left(e^{13}+e^{24}\right),
\end{aligned}\right.
$$

where the $(+)$-sign, resp. (-)-sign, in $d e^{6}$ corresponds to the complex structure $J_{0}^{+}$, resp. $J_{0}^{-}$.

The complex structures $J_{0}^{+}$and $J_{0}^{-}$are then given by $J_{0}^{ \pm} e^{1}=-e^{2}, J_{0}^{ \pm} e^{3}=$ $-e^{4}, J_{0}^{ \pm} e^{5}=-e^{6}$, which means that the real basis $\left\{e^{1}, \ldots, e^{6}\right\}$ is $J_{0}^{ \pm}$-adapted. Therefore, the fundamental form $F$ associated with the $J_{0}^{ \pm}$-Hermitian metric $g=$ $e^{1} \otimes e^{1}+\cdots+e^{6} \otimes e^{6}$ is given by $F=e^{12}+e^{34}+e^{56}$. The structure equations (3.6) imply that

$$
d F=\frac{-2 s}{r} e^{145}+\frac{2 s}{r} e^{235} \mp \frac{2}{r s}\left(e^{135}+e^{245}\right)
$$

For Family II, we first consider the $(1,0)$-basis $\left\{\sigma^{j}\right\}_{j=1}^{3}$ given by

$$
\sigma^{1}=\omega^{1}, \quad \sigma^{2}=\omega^{2}+\frac{i}{2 p^{2}} \omega^{3}, \quad \sigma^{3}=i \omega^{2}-\frac{1}{2 q^{2}} \omega^{3},
$$

where $p^{2}$ and $q^{2}$ are the real, positive and different roots of the polynomial $P(X)=$ $t^{2} X^{2}-s^{2} t^{2} X+\frac{s^{2}}{4}$. In particular,

$$
q^{2}=\frac{s^{2} t^{2}+\sqrt{s^{2} t^{2}\left(s^{2} t^{2}-1\right)}}{2 t^{2}}>0, \quad p^{2}+q^{2}=s^{2}>0, \quad p^{2} q^{2}=\frac{s^{2}}{4 t^{2}}
$$


In terms of $\left\{\sigma^{j}\right\}_{j=1}^{3}$ the fundamental form expresses as $2 F=i\left(r^{2} \sigma^{1 \overline{1}}+p^{2} \sigma^{2 \overline{2}}+q^{2} \sigma^{3 \overline{3}}\right)$. Now, the basis $\left\{e^{1}, \ldots, e^{6}\right\}$ given by

$$
e^{1}+i e^{2}=r \sigma^{1}, \quad e^{3}+i e^{4}=p \sigma^{2}, \quad e^{5}+i e^{6}=q \sigma^{3},
$$

is an adapted basis and the corresponding real structure equations are:

$$
\left\{\begin{aligned}
d e^{1} & =d e^{2}=0 \\
d e^{3} & =\frac{p}{r\left(p^{2}-q^{2}\right)}\left[\mp \frac{1}{p}\left(e^{13}+e^{24}\right) \pm \frac{q}{p^{2}}\left(e^{16}-e^{25}\right)-4 p q\left(q e^{14}+p e^{15}\right)\right], \\
d e^{4} & =\frac{-4 p^{2} q}{r\left(p^{2}-q^{2}\right)}\left[q e^{24}+p e^{25}\right], \\
d e^{5} & =\frac{4 p q^{2}}{r\left(p^{2}-q^{2}\right)}\left[q e^{24}+p e^{25}\right], \\
d e^{6} & =\frac{q}{r\left(p^{2}-q^{2}\right)}\left[\mp \frac{p}{q^{2}}\left(e^{13}+e^{24}\right) \pm \frac{1}{q}\left(e^{16}-e^{25}\right)-4 p q\left(q e^{14}+p e^{15}\right)\right] .
\end{aligned}\right.
$$

Taking into account (3.9), $d F$ is expressed as:

$$
\begin{aligned}
d F=\frac{1}{r\left(p^{2}-q^{2}\right)}[ & \mp\left(e^{134}-e^{156}\right)+4 p q\left(p^{2}+q^{2}\right) e^{145} \pm \frac{p}{q}\left(e^{135}+e^{245}\right) \\
& \mp \frac{q}{p}\left(e^{146}-e^{245}\right)-4 p^{2} q^{2}\left(e^{234}-e^{256}\right) \\
& \left.-4 p q\left(p^{2} e^{235}-q^{2} e^{246}\right)\right] .
\end{aligned}
$$

4. Heterotic string compactifications based on non-nilpotent complex structures. In this section we find explicit solutions of the heterotic supersymmetry equations with non-zero flux and constant dilaton with respect to the Chern connection in the anomaly cancellation condition based on invariant complex structures on nilmanifolds. From the considerations in Section 3 we are led to non-nilpotent complex structures admitting balanced compatible metric, i.e. to a compact nilmanifold $N$ corresponding to the Lie algebra $\mathfrak{n}=(0,0,0,12,23,14-35)$. In Section 4.3 below we provide an explicit realization of $N$ as a compact quotient $\Gamma \backslash K$ of a simply connected nilpotent Lie group $K$ by a lattice $\Gamma$ of maximal rank.

In Section 2 we have proved that any invariant balanced Hermitian structure on $N$ is equivalent to $\left(J_{0}^{ \pm}, F\right)$, where $F$ belongs to the Family I or II of Theorem 2.10. Next we look for solutions of the Strominger system in each family separately.

4.1. Solutions in Family I. We consider first the Family I of balanced Hermitian $\mathrm{SU}(3)$-structures $\left(J_{0}^{ \pm}, F, \Psi\right)$ on $N$. Since $\left\{e^{i}\right\}_{i=1}^{6}$ given in (3.5) is an adapted basis, from (3.7) it follows that the torsion $T$ is given by

$$
T=J_{0}^{ \pm} d F=\frac{2 s}{r} e^{146}-\frac{2 s}{r} e^{236} \pm \frac{2}{r s}\left(e^{136}+e^{246}\right),
$$

which implies, using the structure equations (3.6), that

$$
d T=-\frac{8}{r^{2}}\left(\frac{1}{s^{2}} e^{1234}+s^{2} e^{1256}\right) .
$$

Next we find a large family of $\mathrm{SU}(3)$-instantons for any structure $\left(J_{0}^{ \pm}, F, \Psi\right)$.

Proposition 4.1. For each $\lambda, \mu, \tau \in \mathbb{R}$, let $A_{\lambda, \mu, \tau}$ be the $\mathrm{SU}(3)$-connection defined by the connection 1-forms

$$
\left(\sigma^{A_{\lambda, \mu, \tau}}\right)_{3}^{2}=\left(\sigma^{A_{\lambda, \mu, \tau}}\right)_{5}^{2}=\left(\sigma^{A_{\lambda, \mu, \tau}}\right)_{5}^{4}=\frac{1}{2}\left(\sigma^{A_{\lambda, \mu, \tau}}\right)_{6}^{5}=-\lambda e^{1}-\mu e^{2}-\tau e^{6},
$$




$$
\left(\sigma^{A_{\lambda, \mu, \tau}}\right)_{j}^{i}=\lambda e^{1}+\mu e^{2}+\tau e^{6},
$$

for $1 \leq i<j \leq 6$ such that $(i, j) \neq(2,3),(2,5),(4,5),(5,6)$, and $\sigma_{i}^{j}=-\sigma_{j}^{i}$. Then, $A_{\lambda, \mu, \tau}$ is an $\mathrm{SU}(3)$-instanton and

$$
p_{1}\left(A_{\lambda, \mu, \tau}\right)=\frac{-18 \tau^{2}}{\pi^{2} r^{2} s^{2}} e^{1234}
$$

Proof. Since $\left\{e^{1}, \ldots, e^{6}\right\}$ is an adapted basis for the $\mathrm{SU}(3)$-structure and the connection 1-forms with respect to this basis satisfy $\sigma_{i}^{j}=-\sigma_{j}^{i}$ and

$$
\sigma_{3}^{1}=\sigma_{4}^{2}, \quad \sigma_{4}^{1}=-\sigma_{3}^{2}, \quad \sigma_{5}^{1}=\sigma_{6}^{2}, \quad \sigma_{6}^{1}=-\sigma_{5}^{2}, \quad \sigma_{5}^{3}=\sigma_{6}^{4}, \quad \sigma_{6}^{3}=-\sigma_{5}^{4}, \quad \sigma_{2}^{1}+\sigma_{4}^{3}+\sigma_{6}^{5}=0,
$$

the connection $A_{\lambda, \mu, \tau}$ preserves $F$ and $\Psi$, i.e. it is an $\mathrm{SU}(3)$-connection. A direct calculation, using (3.2) and (3.6), shows that the curvature forms $\left(\Omega^{A_{\lambda, \mu, \tau}}\right)_{j}^{i}$ of the connection $A_{\lambda, \mu, \tau}$ are given by

$$
\begin{aligned}
& \left(\Omega^{A_{\lambda, \mu, \tau}}\right)_{3}^{2}=\left(\Omega^{A_{\lambda, \mu, \tau}}\right)_{5}^{2}=\left(\Omega^{A_{\lambda, \mu, \tau}}\right)_{5}^{4}=\frac{1}{2}\left(\Omega^{A_{\lambda, \mu, \tau}}\right)_{6}^{5}=\frac{-2 \tau}{r s}\left(e^{13}+e^{24}\right), \\
& \left(\Omega^{A_{\lambda, \mu, \tau}}\right)_{j}^{i}=\frac{2 \tau}{r s}\left(e^{13}+e^{24}\right) .
\end{aligned}
$$

Since the 2-forms $\left(\Omega^{A_{\lambda, \mu, \tau}}\right)_{j}^{i}$ satisfy equations (3.1), the connection $A_{\lambda, \mu, \tau}$ is an $\mathrm{SU}(3)$ instanton.

In order to obtain solutions to the anomaly cancellation condition with respect to the Chern connection, we compute first the curvature of $\nabla^{c}$. According to (3.4) and (3.7) the torsion 1-forms $C_{j}^{i}$ of the Chern connection are $C_{2}^{1}=C_{6}^{1}=C_{6}^{2}=C_{4}^{3}=$ $C_{6}^{3}=C_{6}^{4}=C_{6}^{5}=0$ and

$$
\begin{array}{lll}
C_{3}^{1}=C_{4}^{2}=\mp \frac{2}{r s} e^{6}, & C_{4}^{1}=-C_{3}^{2}=-\frac{2 s}{r} e^{6}, & C_{5}^{1}=-\frac{2 s}{r} e^{3} \pm \frac{2}{r s} e^{4}, \\
C_{5}^{2}=\mp \frac{2}{r s} e^{3}-\frac{2 s}{r} e^{4}, & C_{5}^{3}=-\frac{2 s}{r} e^{1} \mp \frac{2}{r s} e^{2}, & C_{5}^{4}= \pm \frac{2}{r s} e^{1}-\frac{2 s}{r} e^{2} .
\end{array}
$$

From (3.3) and the structure equations (3.6) it follows that the non-zero Chern connection 1-forms $\left(\sigma^{c}\right)_{j}^{i}$ are the following:

$$
\begin{array}{ll}
\left(\sigma^{c}\right)_{3}^{1}=\left(\sigma^{c}\right)_{4}^{2}=-\frac{s}{r} e^{5}, \quad & \left(\sigma^{c}\right)_{4}^{1}=-\left(\sigma^{c}\right)_{3}^{2}=\frac{s}{r} e^{6}, \quad \\
\left(\sigma^{c}\right)_{6}^{1}=-\left(\sigma^{c}\right)_{5}^{2}=\mp \frac{1}{r s} e_{5}^{3}, \quad\left(\sigma^{c}\right)_{5}^{3}=\left(\sigma^{c}\right)_{6}^{4}= \pm \frac{1}{r s} e^{2}, & \left(\sigma^{c}\right)_{6}^{3}=-\left(\sigma^{c}\right)_{5}^{4}= \pm \frac{1}{r s} e^{4}
\end{array}
$$

Now, using (3.2) we get the curvature 2-forms $\left(\Omega^{c}\right)_{j}^{i}$ for the Chern connection $\nabla^{c}$ :

$$
\begin{aligned}
& \left(\Omega^{c}\right)_{2}^{1}=-\frac{2}{r^{2} s^{2}}\left(e^{34}+s^{4} e^{56}\right), \\
& \left(\Omega^{c}\right)_{3}^{1}=\left(\Omega^{c}\right)_{4}^{2}=-\frac{1}{r^{2} s^{2}}\left(e^{13}+e^{24}\right), \\
& \left(\Omega^{c}\right)_{4}^{1}=-\left(\Omega^{c}\right)_{3}^{2}= \pm \frac{2}{r^{2}}\left(e^{13}+e^{24}\right)+\frac{1}{r^{2} s^{2}}\left(e^{14}-e^{23}\right), \\
& \left(\Omega^{c}\right)_{5}^{1}=\left(\Omega^{c}\right)_{6}^{2}= \pm \frac{1}{r^{2}}\left(e^{16}-e^{25}\right), \\
& \left(\Omega^{c}\right)_{6}^{1}=-\left(\Omega^{c}\right)_{5}^{2}=\mp \frac{1}{r^{2}}\left(e^{15}+e^{26}\right), \\
& \left(\Omega^{c}\right)_{4}^{3}=-\frac{2}{r^{2} s^{2}}\left(e^{12}-s^{4} e^{56}\right),
\end{aligned}
$$




$$
\begin{aligned}
& \left(\Omega^{c}\right)_{5}^{3}=\left(\Omega^{c}\right)_{6}^{4}=\mp \frac{1}{r^{2}}\left(e^{36}-e^{45}\right), \\
& \left(\Omega^{c}\right)_{6}^{3}=-\left(\Omega^{c}\right)_{5}^{4}= \pm \frac{1}{r^{2}}\left(e^{35}+e^{46}\right), \\
& \left(\Omega^{c}\right)_{6}^{5}=-\left(\Omega^{c}\right)_{2}^{1}-\left(\Omega^{c}\right)_{4}^{3} .
\end{aligned}
$$

Hence, the first Pontrjagin class is represented by

$$
p_{1}\left(\nabla^{c}\right)=-\frac{2}{\pi^{2} r^{4}}\left(e^{1234}+e^{1256}\right)
$$

Therefore, we have proved the following result for the structures belonging to Family I whenever the metric coefficient $s^{2} \geq 1$ :

Theorem 4.2. Let $A_{\lambda, \mu, \tau}$ be the SU(3)-instanton given in Proposition 4.1 with $\tau^{2}=\frac{s^{4}-1}{9 r^{2} s^{2}}$. Then,

$$
d T=2 \pi^{2} \alpha^{\prime}\left(p_{1}\left(\nabla^{c}\right)-p_{1}\left(A_{\lambda, \mu, \tau}\right)\right), \quad \alpha^{\prime}=2 r^{2} s^{2}>0 .
$$

Therefore, $\left(N, J_{0}^{ \pm}, F, \Psi, A_{\lambda, \mu, \tau}, \nabla^{c}\right)$ is a compact solution of the heterotic supersymmetry equations with non-zero flux, non-flat instanton and constant dilaton with respect to the Chern connection in the anomaly cancellation condition, for $r \neq 0, s^{2} \geq 1$, $\tau^{2}=\frac{s^{4}-1}{9 r^{2} s^{2}}$ and for any $\lambda, \mu$.

REMARK 4.3. For $J_{0}^{+}$and $r^{2}=s^{2}=1$ we get the particular solution given in [6]. Notice that for that solution the instanton is flat, so the solutions given in Theorem 4.2 can be thought as a deformation of that particular solution but with instanton having curvature with non-zero trace.

4.2. Solutions in Family II. Now we consider the Family II of balanced Hermitian $\mathrm{SU}(3)$-structures $\left(J_{0}^{ \pm}, F, \Psi\right)$ on $N$. The basis $\left\{e^{i}\right\}_{i=1}^{6}$ given in (3.8) is adapted to the structure and from (3.10) the torsion $T$ is

$$
\begin{aligned}
T=J_{0}^{ \pm} d F & \\
=\frac{1}{r\left(p^{2}-q^{2}\right)}[ & \pm\left(e^{234}-e^{256}\right)+4 p q\left(p^{2}+q^{2}\right) e^{236} \mp \frac{p}{q}\left(e^{136}+e^{246}\right) \\
& \pm \frac{q}{p}\left(e^{235}-e^{136}\right)-4 p^{2} q^{2}\left(e^{134}-e^{156}\right) \\
& \left.-4 p q\left(p^{2} e^{146}-q^{2} e^{135}\right)\right] .
\end{aligned}
$$

From the structure equations (3.9) we get

$$
\begin{aligned}
d T=\frac{-2\left(p^{2}+q^{2}\right)}{r^{2} p^{2} q^{2}\left(p^{2}-q^{2}\right)^{2}}[ & \left(1+16 p^{2} q^{6}\right) p^{2} e^{1234}+\left(1+16 p^{4} q^{4}\right) p q\left(e^{1235}+e^{1246}\right) \\
& \left.+\left(1+16 p^{6} q^{2}\right) q^{2} e^{1256}\right] .
\end{aligned}
$$

From (3.4) and (3.10) it follows that the non-zero torsion 1-forms $C_{j}^{i}$ of the Chern 
connection $\nabla^{c}$ are

$$
\begin{array}{ll}
C_{3}^{1}=\frac{1}{r\left(p^{2}-q^{2}\right)}\left( \pm e^{3} \pm \frac{p}{q} e^{6}\right), & C_{6}^{2}=\frac{1}{r\left(p^{2}-q^{2}\right)}\left(4 p q^{3} e^{3}-4 p^{2} q^{2} e^{6}\right), \\
C_{4}^{1}=\frac{1}{r\left(p^{2}-q^{2}\right)}\left( \pm e^{4} \pm \frac{q}{p} e^{5}+4 p q\left(p^{2}+q^{2}\right) e^{6}\right), & C_{4}^{3}=\frac{1}{r\left(p^{2}-q^{2}\right)}\left(4 p^{2} q^{2} e^{1} \mp e^{2}\right), \\
C_{5}^{1}=\frac{1}{r\left(p^{2}-q^{2}\right)}\left(4 p q\left(p^{2}+q^{2}\right) e^{3} \mp \frac{p}{q} e^{4} \mp e^{5}\right), & C_{5}^{3}=\frac{1}{r\left(p^{2}-q^{2}\right)}\left(4 p^{3} q e^{1} \pm \frac{p}{q} e^{2}\right), \\
C_{5}^{4}=\frac{1}{r\left(p^{2}-q^{2}\right)}\left(\mp \frac{p^{2}+q^{2}}{p q} e^{1}+4 p q\left(p^{2}+q^{2}\right) e^{2}\right), & C_{6}^{1}=\frac{1}{r\left(p^{2}-q^{2}\right)}\left(\mp \frac{q}{p} e^{3} \mp e^{6}\right), \\
C_{3}^{2}=\frac{1}{r\left(p^{2}-q^{2}\right)}\left(4 p^{2} q^{2} e^{3}-4 p^{3} q e^{6}\right), & C_{6}^{4}=\frac{1}{r\left(p^{2}-q^{2}\right)}\left(-4 p q^{3} e^{1} \mp \frac{q}{p} e^{2}\right), \\
C_{4}^{2}=\frac{1}{r\left(p^{2}-q^{2}\right)}\left(4 p^{2} q^{2} e^{4}-4 p q^{3} e^{5} \pm \frac{p^{2}+q^{2}}{p q} e^{6}\right), & C_{6}^{5}=\frac{1}{r\left(p^{2}-q^{2}\right)}\left(-4 p^{2} q^{2} e^{1} \pm e^{2}\right) . \\
C_{5}^{2}=\frac{1}{r\left(p^{2}-q^{2}\right)}\left( \pm \frac{p^{2}+q^{2}}{p q} e^{3}+4 p^{3} q e^{4}-4 p^{2} q^{2} e^{5}\right), &
\end{array}
$$

and therefore, using (3.3) and the structure equations (3.9), the non-zero Chern connection 1-forms $\left(\sigma^{c}\right)_{j}^{i}$ are the following:

$$
\begin{aligned}
& \left(\sigma^{c}\right)_{3}^{1}=\left(\sigma^{c}\right)_{4}^{2}=\frac{1}{2 r\left(p^{2}-q^{2}\right)}\left( \pm e^{3}+4 p^{2} q^{2} e^{4}+4 p^{3} q e^{5} \mp \frac{q}{p} e^{6}\right), \\
& \left(\sigma^{c}\right)_{4}^{1}=-\left(\sigma^{c}\right)_{3}^{2}=\frac{1}{2 r\left(p^{2}-q^{2}\right)}\left(4 p^{2} q^{2} e^{3} \mp e^{4} \mp \frac{q}{p} e^{5}-4 p^{3} q e^{6}\right), \\
& \left(\sigma^{c}\right)_{5}^{1}=\left(\sigma^{c}\right)_{6}^{2}=\frac{1}{2 r\left(p^{2}-q^{2}\right)}\left(-4 p q^{3} e^{3} \pm \frac{p}{q} e^{4} \pm e^{5}+4 p^{2} q^{2} e^{6}\right) \\
& \left(\sigma^{c}\right)_{6}^{1}=-\left(\sigma^{c}\right)_{5}^{2}=\frac{1}{2 r\left(p^{2}-q^{2}\right)}\left( \pm \frac{p}{q} e^{3}+4 p q^{3} e^{4}+4 p^{2} q^{2} e^{5} \mp e^{6}\right), \\
& \left(\sigma^{c}\right)_{4}^{3}=-\left(\sigma^{c}\right)_{6}^{5}= \pm \frac{1}{r\left(p^{2}-q^{2}\right)} e^{2} \\
& \left(\sigma^{c}\right)_{5}^{3}=\left(\sigma^{c}\right)_{6}^{4}=\mp \frac{1}{2 r p q} e^{2} \\
& \left(\sigma^{c}\right)_{6}^{3}=-\left(\sigma^{c}\right)_{5}^{4}=\mp \frac{p^{2}+q^{2}}{2 r p q\left(p^{2}-q^{2}\right)} e^{1} .
\end{aligned}
$$

By (3.2) we get the curvature 2-forms $\left(\Omega^{c}\right)_{j}^{i}$ for the Chern connection $\nabla^{c}$ :

$$
\begin{aligned}
\left(\Omega^{c}\right)_{2}^{1}= & \frac{-1}{r^{2}\left(p^{2}-q^{2}\right)^{2}}\left[\frac{\left(p^{2}+q^{2}\right)\left(1+16 p^{2} q^{6}\right)}{2 q^{2}} e^{34}+\frac{\left(p^{2}+q^{2}\right)\left(1+16 p^{6} q^{2}\right)}{2 p^{2}} e^{56}\right. \\
& \left.+\frac{\left(p^{2}+q^{2}\right)\left(1+16 p^{4} q^{4}\right)}{2 p q}\left(e^{35}+e^{46}\right) \pm 4 p q\left(p^{2}-q^{2}\right)\left(e^{36}-e^{45}\right)\right] \\
\left(\Omega^{c}\right)_{3}^{1}=\left(\Omega^{c}\right)_{4}^{2}= & \frac{-1}{r^{2}\left(p^{2}-q^{2}\right)^{2}}\left[\frac{p^{2}+q^{2}}{4 q^{2}}\left(e^{13}+e^{24}\right) \pm q^{2}\left(3 p^{2}-q^{2}\right)\left(e^{14}-e^{23}\right)\right. \\
& \left. \pm p q\left(3 p^{2}-q^{2}\right)\left(e^{15}+e^{26}\right)-\frac{p^{2}+q^{2}}{4 p q}\left(e^{16}-e^{25}\right)\right] \\
\left(\Omega^{c}\right)_{4}^{1}=-\left(\Omega^{c}\right)_{3}^{2}= & \frac{1}{r^{2}\left(p^{2}-q^{2}\right)^{2}}\left[ \pm\left(2 p^{4}-3 p^{2} q^{2}-q^{4}\right)\left(e^{13}+e^{24}\right)+\frac{p^{2}+q^{2}}{4 q^{2}}\left(e^{14}-e^{23}\right)\right. \\
& \left.+\frac{p^{2}+q^{2}}{4 p q}\left(e^{15}+e^{26}\right) \mp p q\left(p^{2}-3 q^{2}\right)\left(e^{16}-e^{25}\right)\right], \\
\left(\Omega^{c}\right)_{5}^{1}= & \left(\Omega^{c}\right)_{6}^{2}=\frac{1}{r^{2}\left(p^{2}-q^{2}\right)^{2}}\left[\mp p q\left(3 p^{2}-q^{2}\right)\left(e^{13}+e^{24}\right)+\frac{p^{2}+q^{2}}{4 p q}\left(e^{14}-e^{23}\right)\right. \\
& \left.+\frac{p^{2}+q^{2}}{4 p^{2}}\left(e^{15}+e^{26}\right) \pm\left(p^{4}+3 p^{2} q^{2}-2 q^{4}\right)\left(e^{16}-e^{25}\right)\right]
\end{aligned}
$$




$$
\begin{aligned}
& \left(\Omega^{c}\right)_{6}^{1}=-\left(\Omega^{c}\right)_{5}^{2}=\frac{1}{r^{2}\left(p^{2}-q^{2}\right)^{2}}\left[\frac{p^{2}+q^{2}}{4 p q}\left(e^{13}+e^{24}\right) \mp p q\left(p^{2}-3 q^{2}\right)\left(e^{14}-e^{23}\right)\right. \\
& \left.\mp p^{2}\left(p^{2}-3 q^{2}\right)\left(e^{15}+e^{26}\right)-\frac{p^{2}+q^{2}}{4 p^{2}}\left(e^{16}-e^{25}\right)\right], \\
& \left(\Omega^{c}\right)_{4}^{3}=\frac{-1}{r^{2}\left(p^{2}-q^{2}\right)^{2}}\left[\frac{p^{4}-q^{4}}{2 p^{2} q^{2}} e^{12}-\frac{1+16 p^{4} q^{4}}{2} e^{34}-\frac{q^{2}\left(1+16 p^{8}\right)}{2 p^{2}} e^{56}\right. \\
& \left.-\frac{q\left(1+16 p^{6} q^{2}\right)}{2 p}\left(e^{35}+e^{46}\right) \mp 2 p q\left(p^{2}-q^{2}\right)\left(e^{36}-e^{45}\right)\right] \text {, } \\
& \left(\Omega^{c}\right)_{5}^{3}=\left(\Omega^{c}\right)_{6}^{4}=\frac{-1}{r^{2}\left(p^{2}-q^{2}\right)^{2}}\left[\frac{p^{2}+q^{2}}{p q} e^{12}+\frac{p\left(1+16 p^{2} q^{6}\right)}{2 q} e^{34}+\frac{q\left(1+16 p^{6} q^{2}\right)}{2 p} e^{56}\right. \\
& \left.+\frac{1+16 p^{4} q^{4}}{2}\left(e^{35}+e^{46}\right) \pm\left(p^{4}-q^{4}\right)\left(e^{36}-e^{45}\right)\right] \\
& \left(\Omega^{c}\right)_{6}^{3}=-\left(\Omega^{c}\right)_{5}^{4}=\frac{ \pm 1}{r^{2}\left(p^{2}-q^{2}\right)}\left[2 p q\left(e^{34}+e^{56}\right)+\left(p^{2}+q^{2}\right)\left(e^{35}+e^{46}\right)\right], \\
& \left(\Omega^{c}\right)_{6}^{5}=-\left(\Omega^{c}\right)_{2}^{1}-\left(\Omega^{c}\right)_{4}^{3} .
\end{aligned}
$$

Hence, the first Pontrjagin class of the Chern connection is represented by

$$
p_{1}\left(\nabla^{c}\right)=-\frac{2\left(p^{2}+q^{2}\right)}{\pi^{2} r^{4}\left(p^{2}-q^{2}\right)^{2}}\left(\left(p^{2}+q^{2}\right)\left(e^{1234}+e^{1256}\right)+2 p q\left(e^{1235}+e^{1246}\right)\right) .
$$

The following result gives explicit solutions with respect to the Chern connection for the structures belonging to Family II whenever the metric coefficients $s^{2}$ and $t^{2}$ are equal and greater than 1.

TheOREM 4.4. The connection $A_{\lambda, \mu, \tau}$ given in Proposition 4.1 is an $\mathrm{SU}(3)$ instanton for the structures given in Family II if and only if $\tau=0$. With respect to such an instanton, there are solutions to the anomaly cancellation condition if and only if $s^{2}=t^{2}$. In this case,

$$
d T=2 \pi^{2} \alpha^{\prime}\left(p_{1}\left(\nabla^{c}\right)-p_{1}\left(A_{\lambda, \mu, 0}\right)\right)=2 \pi^{2} \alpha^{\prime} p_{1}\left(\nabla^{c}\right), \quad \alpha^{\prime}=2 r^{2}>0 .
$$

Therefore, $\left(N, J_{0}^{ \pm}, F, \Psi, A_{\lambda, \mu, 0}, \nabla^{c}\right)$ is a compact solution of the heterotic supersymmetry equations with non-zero flux and constant dilaton with respect to the Chern connection in the anomaly cancellation condition, for $r \neq 0, s^{2}=t^{2}>1$ and for any $\lambda, \mu$.

Proof. A direct calculation shows that the connection $A_{\lambda, \mu, \tau}$ given in Proposition 4.1 is again an $\mathrm{SU}(3)$-connection, but it is an $\mathrm{SU}(3)$-instanton for a structure in Family II if and only if $\tau=0$, what implies that the curvature vanishes identically. Now, comparing the coefficients of $e^{1235}$ and $e^{1246}$ in $d T$ and $p_{1}\left(\nabla^{c}\right)$, we get that if there is $\alpha^{\prime}$ such that $d T=\alpha^{\prime} p_{1}\left(\nabla^{c}\right)$ then necessarily $\alpha^{\prime}=\frac{r^{2}\left(1+16 p^{4} q^{4}\right) \pi^{2}}{2 p^{2} q^{2}}$. But in this case comparing the coefficients of $e^{1234}$ and $e^{1256}$ we get that $d T$ is a positive multiple of $p_{1}\left(\nabla^{c}\right)$ if and only if $p^{2}-q^{2}=16 p^{4} q^{4}\left(p^{2}-q^{2}\right)$. Since $p^{2} \neq q^{2}$ we conclude that $p^{2} q^{2}=\frac{1}{4}$. Notice that this is equivalent to the metric coefficients $s^{2}$ and $t^{2}$ to be equal, because $p^{2} q^{2}=\frac{s^{2}}{4 t^{2}}$. 
As a consequence of Propositions 2.3 and 2.4, and Theorems 2.10, 4.2 and 4.4, we conclude the existence of many solutions to the Strominger system for any invariant complex structure $J$ on $N$. In fact, such a $J$ is isomorphic to $J_{0}^{+}$or $J_{0}^{-}$, and for each one of these two complex structures there is a four-parametric family of (non-equivalent) balanced Hermitian structures providing solutions: a two-parametric family corresponding to structures in Family I and another two-parametric family corresponding to structures in Family II.

Corollary 4.5. Let $J$ be any invariant complex structure on $N$. Then, there are $\mathrm{SU}(3)$-structures on the compact complex 6-manifold $(N, J)$ solving the heterotic supersymmetry equations with non-zero flux and constant dilaton with respect to the Chern connection in the anomaly cancellation condition.

REMARK 4.6. It has been proved recently [12] that the heterotic supersymmetry and the anomaly cancellation imply the heterotic equations of motion if and only if the connection on the tangent bundle in the $\alpha^{\prime}$ corrections is an SU(3)-instanton. From the explicit expressions of the curvature forms $\left(\Omega^{c}\right)_{j}^{i}$ above, one can see that the Chern connection is never an SU(3)-instanton.

4.3. Explicit realizations. Let us consider the 6-dimensional Lie group

$$
K=\left\{\left(\begin{array}{ccccc}
1 & \frac{1}{2} x_{3} & x_{5} & x_{4} & x_{6} \\
0 & 1 & 2 x_{2} & 2 x_{1} & x_{1}^{2}+x_{2}^{2} \\
0 & 0 & 1 & 0 & x_{2} \\
0 & 0 & 0 & 1 & x_{1} \\
0 & 0 & 0 & 0 & 1
\end{array}\right) \mid x_{i} \in \mathbb{R}\right\}
$$

The left translation $L$ by an element $\left(a_{1}, \ldots, a_{6}\right) \in K$ is given by

$$
\begin{gathered}
L\left(x_{1}, \ldots, x_{6}\right)=\left(a_{1}+x_{1}, a_{2}+x_{2}, a_{3}+x_{3}, a_{4}+a_{3} x_{1}+x_{4}, a_{5}+a_{3} x_{2}+x_{5},\right. \\
\left.a_{6}+a_{5} x_{2}+a_{4} x_{1}+\frac{1}{2} a_{3}\left(x_{1}^{2}+x_{2}^{2}\right)+x_{6}\right) .
\end{gathered}
$$

The following vector fields constitute a basis of left-invariant vector fields on the Lie group $K$ :

$$
X_{1}=\frac{\partial}{\partial x_{1}}+x_{3} \frac{\partial}{\partial x_{4}}+x_{4} \frac{\partial}{\partial x_{6}}, \quad X_{2}=\frac{\partial}{\partial x_{2}}+x_{3} \frac{\partial}{\partial x_{5}}+x_{5} \frac{\partial}{\partial x_{6}}, \quad X_{i}=\frac{\partial}{\partial x_{i}}, \quad i=3,4,5,6 .
$$

Therefore, the Lie algebra of $K$ is generated by elements $X_{1} \ldots, X_{6}$ with non-zero brackets given by

$$
\left[X_{1}, X_{3}\right]=-X_{4}, \quad\left[X_{2}, X_{3}\right]=-X_{5}, \quad\left[X_{1}, X_{4}\right]=\left[X_{2}, X_{5}\right]=-X_{6} .
$$

This Lie algebra is easily seen to be isomorphic to $\mathfrak{n}=(0,0,0,12,23,14-35)$. In fact, with respect to the dual basis of $\left\{X_{1}, \ldots, X_{6}\right\}$, which is given by

$$
\begin{aligned}
& \beta^{1}=d x_{1}, \quad \beta^{2}=d x_{2}, \quad \beta^{3}=d x_{3}, \quad \beta^{4}=d x_{4}-x_{3} d x_{1}, \\
& \beta^{5}=d x_{5}-x_{3} d x_{2}, \quad \beta^{6}=d x_{6}-x_{4} d x_{1}-x_{5} d x_{2},
\end{aligned}
$$

we have that the structure equations of $K$ become $d \beta^{1}=d \beta^{2}=d \beta^{3}=0, d \beta^{4}=\beta^{13}$, $d \beta^{5}=\beta^{23}, d \beta^{6}=\beta^{14}+\beta^{25}$, and thus the relations

$$
e^{1}=\frac{r}{2 s} \beta^{1}, \quad e^{2}=\frac{r}{2 s} \beta^{2}, \quad e^{3}=\beta^{4}, \quad e^{4}=\beta^{5}, \quad e^{5}=\beta^{3}, \quad e^{6}= \pm \frac{1}{s^{2}} \beta^{6},
$$


provide the explicit isomorphism between $\mathfrak{n}$ and the Lie algebra of $K$.

Now, in terms of the coordinates $\left(x_{1}, \ldots, x_{6}\right)$ the balanced Hermitian metrics of the Family I are:

$$
\begin{aligned}
g_{r, s}= & \frac{r^{2}}{4 s^{2}}\left(\left(d x_{1}\right)^{2}+\left(d x_{2}\right)^{2}\right)+\left(d x_{3}\right)^{2}+\left(d x_{4}-x_{3} d x_{1}\right)^{2}+\left(d x_{5}-x_{3} d x_{2}\right)^{2} \\
& +\left[ \pm \frac{1}{s^{2}}\left(d x_{6}-x_{4} d x_{1}-x_{5} d x_{2}\right)\right]^{2} \\
= & \left(\frac{r^{2}}{4 s^{2}}+\left(x_{3}\right)^{2}+\frac{1}{s^{4}}\left(x_{4}\right)^{2}\right)\left(d x_{1}\right)^{2}+\frac{2}{s^{4}} x_{4} x_{5} d x_{1} d x_{2}-2 x_{3} d x_{1} d x_{4}-\frac{2}{s^{4}} x_{4} d x_{1} d x_{6} \\
& +\left(\frac{r^{2}}{4 s^{2}}+\left(x_{3}\right)^{2}+\frac{1}{s^{4}}\left(x_{5}\right)^{2}\right)\left(d x_{2}\right)^{2}-2 x_{3} d x_{2} d x_{5}-\frac{2}{s^{4}} x_{5} d x_{2} d x_{6} \\
& +\left(d x_{3}\right)^{2}+\left(d x_{4}\right)^{2}+\left(d x_{5}\right)^{2}+\frac{1}{s^{4}}\left(d x_{6}\right)^{2} .
\end{aligned}
$$

We can express in a similar way the complex structures $J_{0}^{ \pm}$, the form $\Psi$ and the instantons $A_{\lambda, \mu, \tau}$ in terms of the coordinates $\left(x_{1}, \ldots, x_{6}\right)$. Finally, notice that the discrete subgroup $\Gamma$ can be taken as the subgroup of $K$ consisting of those matrices with integers entries, so that $N=\Gamma \backslash K$.

Acknowledgments. This work has been partially supported through Projects MICINN (Spain) MTM2008-06540-C02-02 and MTM2011-28326-C02-01.

\section{REFERENCES}

[1] K. Becker, M. Becker, J-X. Fu, L-S. Tseng, and S-T. Yau, Anomaly Cancellation and Smooth Non-Kähler Solutions in Heterotic String Theory, Nucl. Phys. B, 751 (2006), pp. $108-128$.

[2] J-M. Bismut, A local index theorem for non-Kähler manifolds, Math. Ann., 284 (1989), pp. 681-699.

[3] S. Chiossi and S. Salamon, The intrinsic torsion of $S U(3)$ and $G_{2}$-structures, Differential Geometry, Valencia 2001, World Sci. Publishing, 2002, pp. 115-133.

[4] L. A. Cordero, M. Fernández, A. Gray, and L. Ugarte, Nilpotent complex structures on compact nilmanifolds, Rend. Circolo Mat. Palermo, 49 suppl. (1997), pp. 83-100.

[5] L. A. Cordero, M. Fernández, A. Gray, and L. Ugarte, Compact nilmanifolds with nilpotent complex structure: Dolbeault cohomology, Trans. Amer. Math. Soc., 352 (2000), pp. 5405-5433.

[6] M. Fernández, S. Ivanov, L. Ugarte, and R. Villacampa, Non-Kaehler heterotic string compactifications with non-zero fluxes and constant dilaton, Commun. Math. Phys., 288 (2009), pp. 677-697.

[7] A. Fino AND G. Grantcharov, Properties of manifolds with skew-symmetric torsion and special holonomy, Adv. Math., 189 (2004), pp. 439-450.

[8] A. Fino, M. Parton, and S. Salamon, Families of strong KT structures in six dimensions, Comment. Math. Helv., 79 (2004), pp. 317-340.

[9] Th. Friedrich And S. Ivanov, Parallel spinors and connections with skew-symmetric torsion in string theory, Asian J. Math., 6 (2002), pp. 303-335.

[10] J-X. FU AND S-T. YAU, The theory of superstring with flux on non-Kähler manifolds and the complex Monge-Ampère equation, J. Differential Geom., 78 (2008), pp. 369-428.

[11] E. Goldstein And S. Prokushrin, Geometric Model for Complex Non-Kähler Manifolds with SU(3) Structure, Commun. Math. Phys., 251 (2004), pp. 65-78.

[12] S. Ivanov, Heterotic supersymmetry, anomaly cancellation and equations of motion, Phys. Lett. B, 685 (2010), pp. 190-196.

[13] M. L. Michelsohn, On the existence of special metrics in complex geometry, Acta Math., 149 (1982), pp. 261-295.

[14] S. Rollenske, Geometry of nilmanifolds with left-invariant complex structure and deformations in the large, Proc. London Math. Soc., 99 (2009), pp. 425-460. 
[15] S. Salamon, Complex structures on nilpotent Lie algebras, J. Pure Appl. Algebra, 157 (2001), pp. 311-333.

[16] A. Strominger, Superstrings with torsion, Nucl. Phys. B, 274 (1986), pp. 253-284.

[17] L. UGarte, Hermitian structures on six-dimensional nilmanifolds, Transform. Groups, 12 (2007), pp. 175-202. 